\title{
Capitalismo, cambios curriculares y formación profesional en Trabajo Social
}

\author{
Capitalism, curriculum changes and traininig in Social Work \\ Fabián GONÓN ORTÍZ \\ Universidad de San Carlos de Guatemala \\ fabiangonon@hotmail.com
}

Recibido: 29/05/2014

Revisado: 30/06/2014

Aceptado: 28/07/2014

Disponible on line: 10/12/2014

\begin{abstract}
Resumen
El papel de la educación superior es de importancia estratégica, dada la necesidad de vinculación con la sociedad y su desarrollo, y sobre todo frente a un proceso capitalista en su expresión neoliberal que profundiza progresivamente las desigualdades sociales. Partiendo de esta realidad y en respuesta a la misma, y pese al momento de crisis de la educación superior pública, el equipo de profesores de la carrera de Trabajo Social del Centro Universitario de Occidente (CUNOC) de la Universidad de San Carlos de Guatemala (USAC) concluye un proceso de Autoevaluación de la Carrera e inicia acciones de Mejoramiento, que incluyen: el fortalecimiento de la investigación para la comprensión y abordaje de la problemática social, la extensión universitaria hacia la sociedad civil y el Estado, la formación y capacitación permanente de profesores y profesoras, la participación de estudiantes en la gestión académico-administrativa, la búsqueda de una estructura académica-administrativa eficaz y eficiente, la innovación del modelo curricular para aumentar la calidad en la formación, los enfoques didácticos y pedagógicos que rebasen la verticalidad y unidireccionaldad en la enseñanza, acceso a redes y sistemas de información y vinculación con entidades académicas nacionales e internacionales, todo ello, con el propósito de mejorar cualitativamente la formación profesional y la intervención de los Trabajadores y las Trabajadoras Sociales; en consecuencia intentar responder a las necesidades sociales de la población en condiciones de exclusión y pobreza, las demandas del mercado ocupacional y el contexto sociopolítico, cultural, económico y ambiental.
\end{abstract}

Palabras clave: capitalismo, autoevaluación, cambios curriculares, rediseño curricular y Trabajo Social.

\begin{abstract}
The role of higher education is of strategic importance given the need to link with society and its development. Furthermore the neoliberal capitalist process is progressively developing social inequalities. Given this reality and in response to it, and despite the time of crisis in public higher education, the teaching staff of the School of Social Work University Center (CUNOC) Western University of San Carlos of Guatemala (USAC) concludes a process of Self-Assessment and initiates actions of Career Enhancement. This activities include: strengthening research for understanding and addressing social issues, university outreach to civil society and the state, permanent pedagogical training of teachers, students participation in academic and administrative management, finding an effective and efficient academic and administrative structure, curriculum innovation model to enhance quality in training, teaching approaches which exceed verticality education, access to networks and information systems and links with national and international academic institutions, all with the purpose of qualitatively improving vocational training and Social Workers intervention; therefore try to respond to the social needs of the population in conditions of exclusion and poverty and the demands of the labor market as well as those of socio-political, cultural, economic and environmental context.
\end{abstract}

Keywords: capitalism, self-assessment, curriculum change, curriculum redesign and Social Work.

Referencia normalizada: Gonón Ortiz, F. (2014): «Capitalismo, cambios curriculares y formación profesional en Trabajo Social». Cuadernos de Trabajo Social, 27(2): 319-328.

Sumario: Introducción. 1. Análisis histórico guatemalteco y cambios curriculares en Trabajo Social. 2. ¿Por qué cambios curriculares en la carrera de Trabajo Social? 3. Autoevaluación. 4. Acreditación. 5. Plan de mejora. 6. Rediseño curricular. 7. Ideas concluyentes. 8. Referencias bibliográficas. 


\section{Introducción}

El capitalismo, como corriente económica, apunta hacia la acumulación de capital y el enriquecimiento privado por encima de las necesidades sociales, «la esencia del capitalismo se centra en la ganancia y la acumulación, en desmedro de la distribución equitativa de la riqueza socialmente producida» (Alayon, 2010, p. 1); en lo político, orienta hacia la reducción de la intervención estatal en materia social o a la reorientación de sus objetivos, limitando las funciones de las políticas sociales a legitimar a las elites de poder y un sistema que genera desigualdad, sin abordar la problemática histórico-estructural. Por el contrario, consolida un Estado que se concreta en crear condiciones para la privatización de las políticas y los servicios sociales, tales como son los casos del seguro social, la salud, la educación, el crédito rural, los servicios básicos, entre otros. De ahí que

La concepción neoliberal de las políticas sociales las considera un medio para alcanzar el bienestar, justicia y la igualdad de oportunidades. En este sentido, su función gravita en la redistribución de ingresos, que permite compensar las desigualdades sociales generadas por el carácter contradictorio del desenvolvimiento del sistema capitalista (Rozas y Fernández, 1988, p. 57).

Sin embargo, esta concepción resulta superficial, puesto que

El Estado no siempre puede impedir la capacidad de respuesta de los sectores dominantes para diluir la eficacia del efecto redistributivo... así como las políticas sociales, por si mismas, no pueden erradicar las injusticias sociales, estas en un sistema capitalista, tienen una matriz estructural (idem).

No puede afirmarse, entonces, que los problemas de desigualdad serán resueltos a través de políticas sociales.

Siendo la política publica el espacio genérico para la intervención del Trabajo Social, resulta imprescindible analizar el Estado y sus Políticas Sociales, los grupos de poder que toman las decisiones macro, la problemática que debiera atender, la actuación de las organizaciones y movimientos sociales frente a ello, así como las contradicciones sociales entre la sociedad y el Estado. Sobre esta realidad multiforme y multicausal deben realizar análisis y critica perma- nente las unidades formadoras de profesionales del Trabajo Social, pues deben incorporar cotidianamente a sus estructuras curriculares y a la formación profesional estos nuevos retos, para que sus egresados respondan a la dinámica de la realidad del país y a los cambios vertiginosos del contexto mundial. «Parece una respuesta obvia considerar que ante las trasformaciones que en la sociedad se van dando, las profesiones deben replantearse sus matrices teóricas, métodos y técnicas, para dar respuestas coherentes a esos cambios» (Cazzaniga, 2007, p. 23). En esta perspectiva se inserta el proceso de autoevaluación y plan de mejoras de la carrera de Trabajo Social y, recientemente, el proceso de transformación curricular. Este autor entiende

Todo cambio curricular como una propuesta que, recuperando los tránsitos realizados (los procesos históricos), se proyecta hacia adelante. Esto quiere decir que si bien el momento histórico en que se realiza una reforma esta presente, un plan de estudios debe tener la capacidad de anticipación y la flexibilidad necesaria para incorporar movimientos permanentes (p. 25).

Las transformaciones sociales, las dificultades respecto de los procesos de enseñanza aprendizaje advertidos, las búsquedas teóricas, las inconsistencias señaladas por los propios egresados, han sido algunos de los aspectos puestos en cuestión para iniciar este proceso de revisión y reformulaciones (idem)

Como también son hechos que justifican transformación curricular en la carrera de Trabajo Social y que se intenta asumir de manera colectiva, cosa nada fácil en el contexto universitario actual.

\section{Análisis histórico guatemalteco y cam- bios curriculares en Trabajo Social}

Los fenómenos socio-políticos tienen siempre un carácter histórico-estructural y por ello el surgimiento y desarrollo de la carrera de Trabajo Social corresponde a cada momento histórico y al contexto nacional y mundial. Visto así, la problemática estructural del país influye permanentemente en la naturaleza del Estado, sus Políticas Sociales y en el desarrollo social, así como en el Trabajo Social. Los cambios curriculares en la carrera de Trabajo Social deben entenderse a la luz del desarrollo histórico del país, pues «Una sociedad, sea mundial o nacional, 
inmersa en un proceso de cambio acelerado en todas las esferas de la vida humana - con todas sus paradojas y contradiccionesexige transformaciones profundas en la organización y operación de la educación en general» (UNIES, 2002, p. 6) y particularmente en la educación superior.

En el caso guatemalteco, tras las relaciones esclavistas y feudales prevalecientes desde el siglo XV, a mediados del siglo XX organizaciones sindicales, estudiantiles, de magisterio, intelectuales y campesinos impulsaron un proceso revolucionario (1944-1954) pro capitalista que viniera a superar las viejas relaciones sociales y fortaleciera las relaciones capitalistas y un incipiente Estado de bienestar. Logros de este periodo fueron el «Decreto 900» que pretendía la expropiación de tierras ociosas y su repartición, el derecho al voto de las mujeres y los analfabetos, el Código de Trabajo, la autonomía universitaria, la autonomía municipal y la creación del Instituto Guatemalteco de Seguridad Social (IGSS), entre otros. Es en este último, marco institucional donde se funda la primera escuela de «Servicio Social», años después incorporada a la Universidad San Carlos como Escuela de Servicio Social (1949). Respecto a la autonomía universitaria, decía Martínez Durán en el Acto de toma de posesión de la Rectoría, el 31 de agosto de 1945. «Os ofrezco mantener incólumes la libertad y la autonomía universitaria, que en hora felicísima para nuestra cultura nos legó la Revolución del 20 de Octubre» (González, 2007, p. 363).

Luego, un proceso contrarrevolucionario (1954) promovido por grupos oligárquicos, el ejército, las transnacionales, como la United Fruit Company (UFCO) y el gobierno de los Estados Unidos derrocan al segundo presidente revolucionario, el coronel Jacobo Arbenz Guzmán, aminorando los logros de la revolución y reconfigurando un nuevo Estado oligárquico y dependiente, dirigido por gobiernos militares autoritarios y represivos durante más de tres décadas; y frente a ello, emergió un nuevo movimiento revolucionario que, sobre la base de una problemática estructural, aglutinaba a sectores campesinos indígenas y a algunos sectores intelectuales y obreros urbanos. Siendo este el marco del periodo del conflicto armado interno (1960-1996).

En estas condiciones sociopolíticas se fundó la Escuela de Servicio Social (1958) en la ciu- dad de Quetzaltenango, como centro regional de la Universidad de San Carlos, comprometida en términos políticos con sectores populares y el propio movimiento revolucionario.

Esfuerzos gubernamentales y de «cooperación» estadounidense, legitimaron su poder, por un lado, utilizando la fuerza y la represión, y por otro lado, a través de políticas sociales, tal es el caso del programa de «Desarrollo de la Comunidad», bajo concepciones «desarrollistas» y motivados por la «revolución verde», como estrategias para enfrentar las condiciones de pobreza de la población rural, según sus autores. Sin embargo, su verdadero propósito fue aminorar la conflictividad sociopolítica, mediatizar el movimiento revolucionario emergente y legitimar a los grupos de poder. En estas condiciones surgió un enfoque «polivalente en la formación de trabajadores sociales» para dar respuesta a las necesidades de la población, pero, sobre todo, a los intereses gubernamentales. Esta formación polivalente de los años 1960 y 1970 incluyó también medicina social, prácticas agronómicas, construcciones rurales y veterinaria, entre otras.

En 1972, intelectuales del Trabajo Social afianzaron la connotación política a la profesión, enriquecida por procesos, como la Teología de la liberación y el movimiento de recoceptualizacion del Trabajo Social en Suramérica. Definieron perfiles, objetivos, principios y funciones profesionales, iniciándose a implementarlos, pese a no haber realizado cambios formales en el currículo. Se avizora aquí un compromiso político del Trabajo Social con los procesos de cambio social e incluso con las «transformaciones estructurales», llegándose hasta niveles de confusión entre lo «profesional» y «la militancia», que más tarde significaron represión y persecución contra la universidad.

En el momento álgido del conflicto se aprueba «el nuevo pensum de Trabajo Social» que inicia su implementación en 1982, a través del cual se quitan las asignaturas señaladas anteriormente y se incluyen otras de filosofía, ciencia política, economía política, antropología y sociología para darle sustento teórico, filosófico y político a la formación. Junto a ello, se fortalece la formación en investigación científica y se consolidan cursos propios del Trabajo Social, influenciados por la reconceptualización sura- 
mericana a través de publicaciones del Centro Latinoamérica del Trabajo Social - CELATSy la Asociación Latinoamericana de Escuelas de Trabajo Social, ALAETS.

El Estado constituía el espacio ocupacional más importante, sin embargo, a finales de los años 1980 inician procesos de reducción de la política social y focalización para resarcir los efectos del conflicto armado interno y compensar los efectos de las políticas de ajuste estructural, tales como los Fondos Sociales y las Secretarías, que fueron la «receta» impuesta por los organismos financieros, como el Banco Mundial y el Fondo Monetario Internacional, a los países latinoamericanos. Las ONG emergen como espacios ocupacionales de mayor importancia, acogidos por la «cooperación internacional» de países como Estados Unidos, Canadá, Alemania y Holanda, entre otros. Un comentario muy ilustrativo sobre esta relación de «cooperación» lo sintetizó una mujer indígena del área rural al decir: "gracias por el apoyo que nos traen, aunque no sé cómo lo tendremos que pagar, porque, nadie regala nada».

El agotamiento del conflicto armado interno, una nueva constitución política de la república, unos gobiernos civiles elegidos con el voto popular y los procesos de negociación de los acuerdos de paz, son las condiciones para la Adecuación curricular (1992) y la Reestructuración curricular (2000). Estos cambios curriculares denotan el interés por mantener la connotación política de la profesión. Sin embargo, en la práctica, se orientaron a atender las demandas más metodológicas y técnicas de nuevos espacios profesionales, las ONG.

Coincide con estos cambios el retiro de una generación de profesores activos durante el conflicto armado interno y con inclinaciones políticas de izquierda. Se incorporan profesores con experiencia profesional en ONG, cuyos intereses se enfocan a una formación de carácter más metodológico y técnico (¿cómo hacer? y ¿con qué hacer?), que político (¿por qué?, ¿para quién hacer? y ¿frente a quién?).

El contexto de estos últimos cambios curriculares se caracteriza por una crisis interna en la Universidad de San Carlos y su politización partidista, su alejamiento de la sociedad y una disminución progresiva en términos de compromiso con la sociedad.

\section{2. ¿Por qué cambios curriculares en la ca- rrera de Trabajo Social?}

«El proceso de globalización económica, la interdependencia mundial y la conformación de bloques regionales constituyen el nuevo contexto internacional en que deben operar las instituciones de educación superior, con todos sus desafíos y oportunidades» (ANUIES, 2000).

Este momento histórico requiere un nuevo paradigma teórico y práctico del Trabajo Social. En primer lugar, porque el proceso neoliberal y la globalización generan desigualdad y profundizan en la pobreza y exclusión social, aparecen nuevos sujetos sociales como los emigrantes, niños y niñas trabajadoras y víctimas de violencia social de género y étnica, ancianos, movimientos sociales en defensa de los recursos naturales; preocupación por el medio ambiente frente al cambio climático y en favor de la vida; grupos diversos reivindicando derechos humanos y específicos.

En segundo lugar, los espacios ocupacionales estatales son sustituidos por las ONG hasta 2010, a partir de entonces el proceso sufre un giro, cuando el Estado inicia a contratar y subcontratar a ONG y empresas por servicios, como un mecanismo para privatizar la política social, tal y como es el caso de los servicios profesionales contratados por el Ministerio de Salud en su programa de extensión de cobertura, o las consultorías contratadas por entidades estatales con fondos de organismos financieros, como el Banco Interamericano de Desarrollo y el Banco Mundial, lo que se proponen es la privatización de la política social más que su institucionalización y fortalecimiento.

En tercer lugar, surgen nuevas demandas por parte de las instituciones empleadoras, de técnicos o profesionales que sepan «hacer» las cosas con una eficiencia y una eficacia que requieren una «calificación distinta» como condición para conseguir empleo. Esto implica menospreciar la importancia del porqué, el para qué y el para quién de la intervención profesional. Son las exigencias de «este modo novedoso y complejo de presencia estatal, a través de organismos paraestatales, las $\mathrm{ONG}$, los organismos internacionales, financieros o humanitarios y las agencias de consultoría (Aquin, 2011, p. 11).

En cuarto lugar, la situación de la Universidad de San Carlos, cuyo compromiso político con la sociedad se sustituye por su crisis acadé- 
mica y su politización partidista. Afectada por el limitado apoyo del Estado, que no le asigna el 5 por ciento del presupuesto que establece la Constitución política, y por el contrario, impulsa la privatización de la educación superior: 14 universidades privadas frente a una Universidad pública. Por otro lado, una propuesta de educación superior limitada a la docencia donde prevalecen enfoques didácticos tradicionales, verticales y bancarios, da poca importancia a la extensión e investigación que, por naturaleza, constituyen mecanismos idóneos para ese vínculo con la sociedad.

En quinto y ultimo lugar, los elementos anteriores obligan a preguntarse: ¿qué Trabajo Social requiere la realidad actual?, ¿qué postura se ha de asumir frente al Estado?, ¿con quiénes trabajar?, desde nuestras intervenciones ¿cómo enfrentar la globalización que impacta por distintas vías?, ¿cómo fortalecer a los sujetos sociales para resolver su problemas inmediatos y avanzar hacia acciones estratégicas políticas?, ¿cómo materializar el aporte del Trabajo Social frente a un problema estructural? ¿qué metodología de intervención será la adecuada? ¿cómo rebasar el simple «hacer»? ¿qué enfoques didácticos y modelos curriculares se han de implementar para mejorar la formación? Estos y otros interrogantes que siguen todavía sin respuesta, constituyen los retos del plan de mejora y rediseño curricular.

\section{Autoevaluación}

En el marco del Sistema Centroamericano de Evaluación y Acreditación de la Educación Superior (SICEVAES) la Universidad de San Carlos de Guatemala, cuya finalidad es promover procesos de autoevaluación de facultades y carreras con fines de mejora y acreditación, los está impulsando como medio para mejorar la formación profesional y el desarrollo de la Universidad. En 2001 las autoridades de la carrera de Trabajo Social tomaron la decisión de iniciar este proceso de autoevaluación, que no avanzó debido a que la Universidad de San Carlos, aunque forma parte del SICEVAES y asumió el compromiso político, ni creó las estructuras ni asignó financiamiento, ni tampoco el tiempo del personal para estas tareas, pues se consideró un trabajo opcional y adicional de cada carrera y de los profesores participantes. Por otro lado, la cultura académica y de trabajo co- tidiano no percibía positivamente este proceso, más bien se consideraba una amenaza, pues requería un esfuerzo extraordinario en ese momento, como también en el futuro. Tampoco hubo un proceso de apropiación del proyecto y de los resultados que se esperaban, lo que mejor se comprendía era la acreditación, pero de manera limitada, pues se consideraba como un «certificado» que calificaría a la carrera de Trabajo Social indefinidamente. De igual forma no se institucionalizó un órgano rector de estos procesos en el Centro Universitario de Occidente (CUNOC), porque desde la Universidad de San Carlos central era difícil apoyar a los centros regionales. Por esta razón, los enfoques y criterios manejados para ello eran muy distintos a nivel de autoridades del CUNOC y profesores de la carrera de Trabajo Social. Finalmente, la inexperiencia en procesos de esta naturaleza generó una percepción más de «obligación» que de «oportunidad».

En 2010 las autoridades de la carrera de Trabajo Social retoman esta iniciativa por «presiones» de la Universidad San Carlos, se elabora un plan de trabajo y se asigna al Departamento de Investigaciones en Trabajo Social (DITSO) para que, a tiempo parcial, tres profesores inicien esta labor. Un elemento positivo fue que el director de esta unidad poseía experiencias similares en universidades mexicanas. Además, se estableció un acuerdo con profesores de planta (10) para su involucramiento, asignando responsabilidades y tiempos. Durante este primer año, el equipo se concentró en conocer el proyecto de autoevaluación, los factores y criterios, y los instrumentos (cuestionarios) propuestos por la comisión de Autoevaluación de la Universidad de San Carlos, que fueron en tres ocasiones reformulados y adaptados a los requerimientos específicos de la carrera de Trabajo Social.

En 2011 se organizaron equipos de trabajo, con 2 ó 3 profesores, que procedieron a recolectar la información a través de cuatro cuestionarios dirigidos a: estudiantes, profesores, egresados y directores de instituciones empleadoras. Se obtuvo información con la totalidad de estudiantes de la carrera de Trabajo Social y profesores de plantilla y temporales.Para el caso de egresados y empleadores hubo necesidad de visitar o contactar a profesionales y empleadores de todo el país. Sin embargo, la recolección de 
información se enfatizó en la región de cobertura del Centro Universitario de Occidente, es decir, en los departamentos de Totonicapán, Sololá, Huehuetenango, Quiché, San Marcos, Retalhuleu, Suchitepéquez y Quetzaltenango, una región caracterizada por población indígena, rural y en condiciones de pobreza. Algo de destacar, es que algunos egresados de la carrera de Trabajo Social son profesores en la escuela del campus central de la Universidad de San Carlos y en la mayoría de los centros regionales.

Durante el año 2012 se procedió a sistematizar la información cuantitativa (tabular y clasificar), se reorganizaron de nuevo los equipos de profesores para elaborar informes preliminares de acuerdo a cada uno de los 9 factores siguientes: desarrollo curricular, estudiantes, profesores y personal de apoyo, gestión académica, recursos, investigación y desarrollo, extensión y vinculación, factores de egresados y empleadores. Para contar con suficiente información, sobre todo cualitativa, se procedió a realizar talleres, seminarios y reuniones con los cuatro grupos ya mencionados para analizar la información estadística. De esta manera se concluyó el primer informe preliminar que, además de los resultados de la autoevaluación, incluía un Plan de Mejora y anexos (datos estadísticos), concretándose en un documento de 236 páginas.

Se elaboró un informe ejecutivo de 25 páginas como instrumento para la validación, con los cuatros grupos de informantes miembros de la comunidad educativa, es decir: estudiantes, docentes, egresados y empleadores. Durante el año 2012 y todo el año 2013 fue un espacio para la validación del informe de autoevaluación y el plan de mejoramiento; inicialmente, la validación se realizó con un grupo de estudiantes (25), con egresados (18), con empleadores (12) y con todo el personal de planta de la carrera de Trabajo Social (10). Para fortalecer el proceso de validación, se realizaron talleres de socialización nuevamente con el personal, pero agregados los profesores alternos y auxiliares (20), así como con un grupo mucho más representativo de estudiantes ( 5 por semestre, 30 en total) y finalmente con autoridades de la carrera de Trabajo Social, de la División de Humanidades y Ciencias Sociales y autoridades del Centro Universitario de Occidente (la Directora General y la Directora Académica). El propósito era socializar, sensibilizar y enriquecer continuamente el informe, como también que todas las personas se apropiaran del proceso y asumieran compromiso «político» para impulsar el seguimiento a las actividades posteriores, especialmente la implementación del plan de mejora y la acreditación de la carrera, pues estas actividades, además de los esfuerzos del equipo, requieren recursos financieros y el respaldo de las autoridades. Se documentaron todos los eventos con sus respectivas actas y minutas, en las cuales quedaron plasmados acuerdos y compromisos.

Después de cuatro correcciones se cuenta con el informe final de autoevaluación y el plan de mejora contenido en 115 páginas.

Al identificar que el estudiantado en su totalidad no había tenido una participación activa en este proceso, se decidió realizar un seminario con estudiantes (300 aproximadamente), para que se apropiaran de los resultados obtenidos y progresivamente se fueran involucrando en las tareas siguientes, es decir,. en la implementación del plan de mejora y acreditación de la carrera.

El informe de autoevaluación indicaba que, a pesar del contexto neoliberal y globalizante, con un Estado limitado en su intervención social y con la Universidad San Carlos en una situación de crisis política y académica, la carrera de Trabajo Social tiene potencialidades con las cuales puede enfrentar retos como los siguientes:

1. La formación profesional relativamente distanciada de las exigencias y retos de la sociedad, el contexto, el mercado ocupacional y sobre todo de la realidad social.

2. Distanciamiento entre la unidad formadora (carrera de Trabajo Social ) con sus egresados y los espacios ocupacionales, que impide la proyección y fortalecimiento de la intervención, así como la ampliación de espacios profesionales. Como también experiencias y vivencias que realimenten el desarrollo de la profesión y la formación profesional.

3. Las tareas de investigación limitadas a fines de promoción académica, no en función de las necesidades y problemáticas sociales.

4. La extensión universitaria limitada a las «prácticas» a nivel técnico y licenciatura en instituciones. Pero no existen otras acciones permanentes que garanticen su vinculación con organizaciones sociales y redes institucionales.

5. Se carece de mecanismos definidos para la investigación y el desarrollo académico per- 
manentes. El aprender a aprender es solamente una idea, no una práctica.

6. La estructura administrativa y de gestión académica es insuficiente y centralizada y la participación política estudiantil mínima. $\mathrm{La}$ formación requiere enriquecimiento con nuevas estrategias, enfoques de enseñanza, metodologías, sistemas de información y redes.

7. Carencias en términos de infraestructura, laboratorio de cómputo, centro de información y documentación, oficina de registro e información, aulas y salas de trabajo, despachos de profesores y equipo, entre otras.

Retos que obligan a potenciar al máximo los 50 años de experiencia institucional en la formación profesional de trabajadores sociales y los recursos con que cuenta la carrera de Trabajo Social. Entre ellos, la experiencia de egresados ubicados en centros formadores y diversos espacios ocupacionales del país, profesores formados en su mayoría a nivel de Máster, experiencia en términos de las prácticas vistas como extensión, departamento de investigaciones con 10 años de trabajo, algunos profesores vinculados a organizaciones sociales e inicio de tareas de intercambio académico entre otros.

Es de resaltar que el grupo de profesores se apropia cada vez más de estos procesos de autoevaluación y mejora permanentes, continuos y participativos, y hay más comprensión de que los cambios curriculares se refieren más que a modificar un documento formal a transformaciones en las actitudes de los profesores, de los estudiantes, de las autoridades y al aporte de egresados. Al respecto, Alba América Acevedo, desde el Departamento de Desarrollo Académico del campus central de la Universidad de San Carlos, en una de sus recientes visitas manifestó: «es interesante cómo se han apropiado del proceso, veo que se están involucrando e involucrando a los demás y poco a poco están cambiando»

\section{Acreditación}

El propósito inicial de los profesores fue la acreditación de la carrera, más que su mejora, idea que se ha revertido; ahora la prioridad es mejorar para alcanzar la acreditación. Una de las limitaciones iniciales, y que progresivamente se fue superando, era considerar la acreditación como un certificado que podía obtenerse auto- máticamente y cuya utilidad era indefinida. Algunos, equivocadamente, consideraban que, al realizar el proceso de autoevaluación y lograr la visita de pares académicos, prácticamente se obtenía la acreditación, cuando en realidad es un proceso permanente, un proyecto de vida universitaria, donde la acreditación y reacreditación son continuas en una perspectiva de mejora progresiva: es, pues, la institucionalización de la actualización constante y la construcción de una cultura de análisis, reflexión y cambio incorporado a la cotidianidad de la carrera de Trabajo Social.

Uno sistema para la acreditación de las entidades académicas certificadas es el Sistema Nacional de Evaluación de Estudios Superiores (SINAES) de Costa Rica; al analizar la guía que utilizan para acreditación de carreras, particularmente los 348 criterios, y contrastarla con la realidad de la carrera de Trabajo Social, esta última solo puede alcanzar un 18 por ciento de esos criterios de evidencia; si se trabajara a conciencia en la documentación de otros criterios podría alcanzar un 37 por ciento más a corto plazo (de 1 a 2 años), llegando a un total del 55 por ciento. Esto significa, que la implementación del plan de mejora resulta indispensable para superar este porcentaje y lograr la acreditación.

\section{Plan de mejora}

En base a la comprensión general de la carrera de Trabajo Social a través del Informe de autoevaluación se definió el plan de mejora en cuyo contenido se destacan las acciones siguientes: 1) Rediseño curricular a través del modelo por competencias, 2) Fortalecimiento del Departamento de Investigación en cuanto a estrategias, estructura y recurso humano, 3) Creación del Departamento de Extensión y vinculación, donde la práctica sea uno de sus componentes, 4) Implementación de Diplomaturas y Maestrías en Trabajo Social y afines, Intercambio académico permanente, 5) Formación del profesor/investigador o investigador/profesor 6) Constitución de Registro e Información y atención estudiantil, laboratorio de computo y espacio para videoconferencias, centro de gestión de la información documental y electrónica, 7) Incorporación de estudiantes en la estructura de gestión académico administrativa, 8) Gestión académica y administrativa descentralizada, 
eficiente y eficaz y 9) Mejora de infraestructuras, mobiliario y equipos. Todo ello, en búsqueda de calidad e innovación profesional, congruencia y pertinencia profesional con las necesidades del país y mayores y mejores espacios profesionales.

Este plan de mejora se aprobó formalmente en noviembre del 2013, en una reunión de profesores donde se acordó: iniciar a implementar el plan de mejora, elaborar un plan operativo para el año 2014 y seguir con la misma estructura organizativa por áreas para continuar con la cotidianidad de la carrera, pero a la vez, iniciar las actividades del plan de mejoras. También se acordó como prioritario iniciar con el proceso de rediseño curricular, alrededor del cual deben planificarse todas las actividades de la carrera de Trabajo Social.

En paralelo con los procesos de elaboración y validación del plan de mejora, se avanzó en su ejecución; entre lo más destacable está la formación de profesores a través de diplomaturas en temáticas como: «Derechos Humanos», «Conflictividad Social y Prevención de Violencia», «Gestión Territorial y Perspectivas de Paz», que fueron coordinadas con una expresión de la sociedad civil, denominada «Espacio de Diálogo», y coordinada con la Oficina de los Derechos Humanos del Arzobispado de Guatemala (ODAHG), así como mediante otros eventos virtuales. Además, se inició la vinculación de profesores a la Mesa Occidental del Agua (MOA) un espacio de organizaciones e instituciones que trabajan alrededor del tema de agua, el medioambiente y los recursos naturales, desde donde se realizó el III Congreso del Agua: el Estado, sus políticas públicas y las demandas de la sociedad civil; a través de esta coordinación, también surgió la necesidad de formulación de la Maestría en Ciudadanía para la Gestión de los Recursos Naturales que cuenta con una propuesta y ahora se discute internamente para gestionar su aprobación; del mismo modo, surge la necesidad de crear un Centro de Documentación e Información que ahora ha iniciado la carrera de Trabajo Social.

Las políticas de investigación también se han discutido con profesores, se han actualizado y se han establecido como prioridades cinco líneas: neoliberalismo, el Estado y la sociedad, las políticas sociales, la problemática de pobreza y exclusión social de nuestros sujetos de acción, las organizaciones y movimientos sociales y enfoques, metodologías y técnicas cualitativas y participativas del Trabajo Social. Estas, aunque ya guían la investigación y sistematización, deben aún incorporarse en el rediseño curricular para su institucionalización. Además, se han desarrollado modalidades de investigación y han surgido «grupos de investigación» en torno a temáticas especificas.

Se ha sometido a discusión el proyecto de «Extensión» donde se considera una estrategia de prácticas, y además, programas de voluntariado, vinculación de profesores con organizaciones, movimientos sociales y sector público, generación de redes de trabajo, programas de formación a dirigentes de base, vinculaciones en base a investigación para la incidencia etc. Extensión e investigacion obligan ahora a crear estrategias de gestión financiera interna a la Universidad de San Carlos y de organismos externos, pues los recursos para estas actividades son limitados.

Dentro de la Universidad se han establecido vínculos, al igual que se buscan ahora espacios de intercambio académico con universidades como Innsbruck de Austria, UNAM de México y Universidad Complutense de España.

De importancia estratégica es la formación de profesores, mediante programas de doctorado, master y cursos de postgrado, cursos virtuales, cursos cortos, intercambios con universidades nacionales e internacionales, aprendizaje de inglés, aprendizaje de idiomas locales y gestión de información electrónica, entre muchos otros, tal y como este proceso parece generar necesidades de formación.

\section{Rediseño curricular}

Una de las prioridades dentro del plan de mejora es el rediseño curricular, que es un hecho necesario dado que la actual reestructuración curricular entró en vigencia en 2001, hace ya 13 años, y como consecuencia no responde ya a los retos que plantea el nuevo contexto, a las nuevas demandas del mercado ocupacional y a las necesidades de la sociedad, particularmente, de aquellos grupos sociales en condiciones de pobreza y exclusión social, sujetos sociales del Trabajo Social guatemalteco.

Sustantivo para iniciar el proceso de rediseño curricular es el acuerdo tomado con todos los profesores y la decisión de las autoridades del 
Centro Universitario de Occidente de nombrar una comisión integrada por coordinadores de área (mandos medios) y el apoyo de las autoridades de la carrera de Trabajo Social y de la División de Humanidades. Teniendo esto como base, se ha procedido a elaborar un plan de trabajo que involucra y responsabiliza a todos, organiza subcomisiones, asigna resultados, tiempos y recursos disponibles.

El esfuerzo principal es el rediseño curricular y, para complementarlo, la comisión de Desarrollo Académico, ha diseñado una serie de eventos (conferencias, foros, paneles, seminarios, intercambios) de formación y actualización de profesores y estudiantes en torno al papel de la Universidad en la sociedad, ante la realidad nacional y sus nuevas expresiones, los enfoques didácticos y pedagógicos innovadores, la transformación y el cambio curricular en el nuevo contexto, así como los diversos modelos curriculares existentes. El mismo rediseño curricular se realiza en el marco del «Seminario de Rediseño Curricular» para lo cual existen reuniones de subcomisiones semanales, y luego, reuniones mensuales para socializar, colectivizar y enriquecer resultados y avances.

En esta misma lógica, la comisión de SICEVAES vincula sus acciones en torno al rediseño curricular. Por ejemplo: este proceso de rediseño curricular debe, en lo posible, responder a los 9 factores y los 348 indicadores que plantea la guía para la acreditación de carreras, es decir, el rediseño mismo debe atender explicita o implícitamente las necesidades y los requisitos para la acreditación. También, es de destacar el proyecto de «Homologación» que se ha iniciado entre carreras de Trabajo Social de la Universidad de San Carlos y que, además de constituirse en un requisito para la acreditación, facilita la movilidad estudiantil y docente, que ha requerido análisis, discusión y acuerdos en conjunto. Recientemente, inició un proceso para crear un «sistema categorial» que sustente la homologación, esta es una buena oportunidad para generar el debate teórico conceptual del Trabajo Social. Interesante ver esa relación dialéctica entre plan de mejoras, el rediseño curricular, la acreditación y homologación y la forma en que se enriquecen y se complementan.

En la Universidad San Carlos, campus central, se movilizan los recursos humanos, especialmente de unidades como el Departamento de Desarrollo Académico (DDA), Dirección General de Docencia (DIGED) y el Departamento de Actualización y Organización Curricular (DAOC), Así mismo, este proceso también ha obligado a socializar, compartir y establecer coordinaciones y alianzas con otras carrera dentro del mismo Centro Universitario de Occidente: por ejemplo, la División de Ciencia y Tecnología, que inicia la implementación de nuevas carreras sobre la base de un modelo por competencias, o la carrera de Pedagogía que en 2013 inició la implementación de su rediseño curricular con enfoque holístico.

\section{Ideas concluyentes}

Una realidad mundial neoliberal y global determinante e influyente en el Estado y la sociedad, requiere trasformaciones profundas en la educación superior y particularmente cambios curriculares, para asegurar que

La finalidad de la universidad no puede ser la de hacer que una clase privilegiada se reproduzca indefinidamente (...) ni Bastilla de Casta, ni foco de insurrección, la Universidad ha sido creada para la elaboración crítica del conocimiento, para la transmisión crítica del conocimiento, para la puesta en tela de juicio permanente del conocimiento (García-Ruiz, 1998, p. 142).

De cara a una realidad desigual, inequitativa y excluyente que requiere propuestas innovadoras, practicas y pertinentes en cuanto al género y la realidad étnica.

La Universidad de San Carlos de Guatemala y sus unidades académicas, entre ellas la carrera de Trabajo Social, no dejan de ser un reflejo de la sociedad, con sus limitaciones y bondades. Entendido esto, en contexto de adversidades y fortalezas se ha iniciado un proceso de autoevaluación, de formulación de un plan de mejora y de implementación, que incluye transformaciones curriculares que se materializan en el «rediseño curricular», lo que conlleva mejoras de la formación profesional, capacidades de investigación y vinculación y extensión hacia la sociedad, con el propósito de contar con profesionales abiertos al aprendizaje, críticos y propositivos.

La participación, el diálogo, el análisis y los consensos han hecho que un equipo sea protagónico en la autoevaluación y mejora de esta unidad académica, quizá no con los mismos niveles de compromiso y apropiación, pero sí va 
camino de ello, en búsqueda de una mejor formación profesional en Trabajo Social, en consecuencia una sustantiva intervención profesional. Esta debe "comprometerse» con las necesidades de la sociedad, en países como Guatemala identificados con aquella población victima de exclusión social y pobreza.

Este proceso ha conllevado, apropiación y entendimiento de la realidad de esta unidad académica y su contexto, se han tomado decisiones conjuntas y consensuadas que orientan acciones de mejoramiento, pero sobre todo, se ha avanzado hacia acciones, quizá pequeñas pero muy significativas, en aras de lo que se aspira en un contexto adverso. Paso a paso se espera involucrar más a todos los docentes, y muy en especial, a estudiantes que son o deben ser una fuerza viva en toda entidad académica, a los egresados que con su ejercicio y experiencia pueden realimentar este esfuerzo por cambiar, así como enriquecer su formación. Se necesita una mayor apertura también frente a la sociedad y otras entidades académicas, más sinergias con actores comprometidos con el cambio social.
Este proceso de mejoras ha obligado a repensar el Trabajo Social sin encontrar respuestas definitivas; su naturaleza, su espacio y papel, paradigmas y perspectivas, exigen un repensar teórico, epistemológico, filosófico y metodológico, para rebasar aquellas visiones y prácticas tradicionales que aún caracterizan a nuestra profesión: paternalismo, asistencialismo y filantropía. Y luego, con las respuestas que se encuentren, permear una mejor formación. Espacios creados a través de una serie de talleres, reuniones, seminarios y conversatorios en los cuales progresivamente se ve más compromiso.

Lo importante es ver alternativas, opciones, caminos. La cuestión es saber desde qué proyecto de futuro se construye hoy día el conocimiento de lo social. La responsabilidad es la misma que en el pasado cuyo recuerdo nos vuelve pesimistas. Salgamos de la crisis menor para enfrentar otra, la que proclaman el caos en la ciencia misma. Desde un cierto escepticismo será posible construir certidumbres. Esa es la tarea para la generación que nació con internet. Es decir, que ya empezó a caminar (Torres-Rivas, 2001, p.42).

\section{Referencia bibliográficas}

Alayon, Norberto (2010). Política Social y Trabajo Social. (Ponencia Presentada en el Encuentro Regional, México, Centro América y El Caribe. Universidad de San Carlos de Guatemala).

Aquin, Nora (2011). Reconfiguraciones del trabajo social en el contexto de la globalización. Revista Escuela de Trabajo Social. México: Universidad Nacional Autónoma de México.

Asociación Nacional de Universidades e Instituciones de Educación Superior. (2000). La Educación Superior en el Siglo XXI, líneas estratégicas de desarrollo. Una propuesta. México: ANUIES.

Cazzaniga, Susana (2007). Reformulación del plan de estudios: contribuciones para el debate. (Documento de trabajo) Facultad de Trabajo Social UNER. Espacio Editorial, Argentina.

Carrera de Trabajo Social. (2013). Informe de Autoevaluación y Plan de Mejoramiento. (Informe preliminar). Quetzaltenango: Centro Universitario de Occidente de la Universidad de San Carlos de Guatemala.

Fernández, Arturo y Rozas, Margarita (1988). Políticas Sociales y Trabajo Social. Buenos Aires: Editorial Hvmanitas.

García-Ruiz, Jesús (1998). La Universidad a las puertas del tercer milenio. Guatemala: Ediciones Asociación Maya de Estudiantes Universitarios.

Gonzales Orellana, Carlos (2007). Historia de la Educación en Guatemala. (7ª . Edición). Guatemala: Editorial Universitaria USAC.

Torres-Rivas, Edelberto (2001). Acerca del pesimismo en las ciencias sociales. Guatemala: Facultad Latinoamericana de Ciencias Sociales. 